\title{
Association of DI02 Thr92Ala Polymorphism With Pediatric Obesity In Japanese Children: A Case Control Study
}

\section{Takeshi Ota}

Kyoto Prefectural University of Medicine: Kyoto Furitsu Ika Daigaku Jun Mori ( $\nabla$ jun1113@koto.kpu-m.ac.jp )

Kyoto Prefectural University of Medicine: Kyoto Furitsu Ika Daigaku https://orcid.org/0000-0002-12711577

\section{Hirotsugu Yasuda}

Kondo Photo Incorporated

\section{Mihoko Yamaguchi}

Kyoto Prefectural University of Medicine: Kyoto Furitsu Ika Daigaku

\section{Satoshi Miyagaki}

Kyoto Prefectural University of Medicine: Kyoto Furitsu Ika Daigaku

\section{Yasuhiro Kawabe}

Kyoto Prefectural University of Medicine: Kyoto Furitsu Ika Daigaku

\section{Yusuke Tsuma}

Kyoto Prefectural University of Medicine: Kyoto Furitsu Ika Daigaku Hidechika Morimoto

Kyoto Prefectural University of Medicine: Kyoto Furitsu Ika Daigaku Keiichi Shigehara

Kyoto Prefectural University of Medicine: Kyoto Furitsu Ika Daigaku

\section{Shota Fukuhara}

Kyoto Prefectural University of Medicine: Kyoto Furitsu Ika Daigaku

\section{Kazuki Kodo}

Kyoto Prefectural University of Medicine: Kyoto Furitsu Ika Daigaku

\section{Satoru Sugimoto}

Kyoto Prefectural University of Medicine: Kyoto Furitsu Ika Daigaku

\section{Kitaro Kosaka}

Kyoto Prefectural University of Medicine: Kyoto Furitsu Ika Daigaku

\section{Hisakazu Nakajima}

Kyoto Prefectural University of Medicine: Kyoto Furitsu Ika Daigaku

\section{Hajime Hosoi}

Kyoto Prefectural University of Medicine: Kyoto Furitsu Ika Daigaku 
Research

Keywords: obesity, BAT, DIO2, UCP1, $\beta 3$ adrenergic receptor

Posted Date: December 28th, 2020

DOl: https://doi.org/10.21203/rs.3.rs-131077/v1

License: (c) (i) This work is licensed under a Creative Commons Attribution 4.0 International License. Read Full License 


\section{Abstract}

\section{Background}

Genetic factors play a critical role in the onset and progression of obesity. Brown adipose tissue (BAT) activity is also critical for adiposity. The objective of this study was to evaluate the prevalence and effects of gene polymorphisms related to BAT in pediatric obesity.

\section{Methods}

A case-control study with 270 non-obese and 86 obese children was performed. All participants underwent genotyping of type 2 deiodinase (DIO2) Thr92Ala (rs225014), uncoupling protein 1 (UCP1) -3826 A/G (rs1800592), and $\beta 3$ adrenergic receptor ( $\beta 3 A R)$ Trp64Arg (rs4994).

\section{Results}

In the case-control study, the prevalence of the homozygous Ala/Ala allele of the DIO2 gene in the obese group was $15.1 \%$ versus $6.3 \%$ in the non-obese group, resulting in an odds ratio (OR) of 3.393 (95\% confidence intervals [CI], 1.498-7.687, $P=0.003$ ). The genotype distribution of UCP1-3826 A/G and B3AR Trp64Arg did not significantly differ between the obese and the non-obese groups, and the ORs were $1.831(95 \% \mathrm{Cl}, 0.955-3.512, P=0.069)$ and $0.819(95 \% \mathrm{Cl}, 0.263-2.555, P=0.731)$, respectively.

\section{Conclusions}

Our results indicate that the homozygous Ala/Ala allele of the DIO2 gene is associated with an increased risk of obesity in children.

\section{Background}

Obesity is a complex medical disease due to multiple factors, including genetic susceptibility and environmental and lifestyle factors, and is associated with an increased risk of various diseases, including cardiovascular disease, type 2 diabetes mellitus, and cancer (1). Obesity is increasing globally, even in children. Pediatric obesity tends to lead to continued obesity in adulthood; thus, the pediatric obesity epidemic is considered one of the most important health issues. Genetic factors in obesity account for $40-70 \%$ (2) and some single-nucleotide polymorphisms (SNPs) are associated with pediatric obesity (3); thus, it is important to evaluate the genetic background in pediatric obesity.

Recently, brown adipose tissue (BAT) has been identified as a novel target of obesity, as it increases energy expenditure by non-shivering thermogenesis. BAT exists even in adulthood, but is highly present in childhood. Enhancing BAT could be an attractive strategy for combating pediatric obesity (4-6). Therefore, it is highly likely that BAT activity is critical for adiposity, and the polymorphism of genes related to BAT might cause obesity in childhood. 
Type 2 deiodinase (DIO2) converts thyroxine to triiodothyronine (T3), a biologically active form. Thyroid hormone (TH) stimulates thermogenesis via the induction of mitochondrial uncoupling protein 1 (UCP1) in BAT. The thermogenic effect of BAT in response to TH is caused by the synergistic interaction with the sympathetic nervous system (SNS) through the $\beta 3$ adrenergic receptor ( $\beta 3 A R$ ). TH receptors, both $a$ and $\beta$ subunits, are expressed in BAT. Synergism between TH and SNS causes an effect via TRa, and the transcription of UCP1 is upregulated by TR $\beta$ (7). In addition, increased tissue levels of T3 amplify the SNS effects, including UCP1 gene transcription.

In the present study, we focused on the following three genes: DIO2, UCP1, and $\beta 3 A R$. These genes are key molecules in BAT thermogenesis. In fact, some studies have demonstrated that these genes are associated with the pathogenesis of adulthood obesity (8-11). However, few studies have reported the association between these genes and pediatric obesity. Therefore, we examined the effects of DIO2 Thr92Ala, UCP1-3826 A/G, and B3AR Trp64Arg polymorphism on pediatric obesity.

\section{Methods}

Subjects

This study was approved by the institutional review board of Kyoto Prefectural University of Medicine. According to Japanese guidelines, individuals with a percentage of overweight (POW) $\geq 20 \%$ are classified as obese (12). POW, which is the modified weight-for-height method, is widely used as a surrogate marker of childhood obesity in Japan (13). POW is calculated using the following formula:

POW $(\%)=100 \times($ measured weight - standard weight $) /$ standard weight

Japanese standard weight is the age- and sex-specific weight for height, which is based on data from the Annual Report of School Health Statistics 2000 by the Ministry of Education, Culture, Sports, Science, and Technology, Japan. POW is reported to be a more appropriate method than BMI \% for school-age children. A POW of $20 \%$ is equivalent to approximately the 90 th BMI \% of children with average height and weight, and the criteria for obesity are defined as POW $\geq 20 \%$ ( $\geq 120 \%$ of the standard weight) (14). For nonobese children (NOB), study participants were recruited during an annual medical check-up at a junior high school in Kyoto. The NOB group did not include children with underlying conditions. Informed consent was obtained from 288 children and their parents. Eighteen children were excluded from the NOB group due to a high POW. These children were included in the obese children group (OB). Eventually, 270 children were enrolled in the NOB group. The median (range) age was 13.5 years (12.1-15.2). For OB, we selected 68 obese children who visited our outpatient clinic. We obtained informed consent for these children from their parents as well. Finally, we enrolled 86 children as OB ( 18 children as above were added to this group) (Fig 1). The median (range) age was 11.1 years (4.6-17.5) (Table 1). POW is unique to Japan; thus, the Rohrer index was used to verify that the same results could be obtained using global obesity standards. The presence or absence of obesity was determined using the Rohrer index, and the same analysis was performed. The Rohrer index was determined in kilograms per cubic meter, and the 
criteria for obesity were defined as Rohrer's index $\geq 145$. Based on the Rohrer index, we classified 265 and 91 children into the NOB and OB groups, respectively (Table 2).

Genotyping

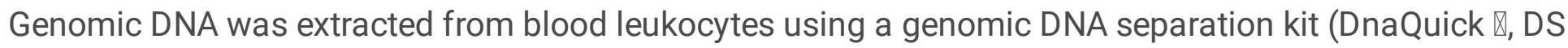
Pharma Biomedical, Osaka, Japan). We analyzed the following polymorphisms of three genes; DIO2 Thr92Ala (rs225014), UCP1-3826 A/G (rs1800592), and ß3AR Trp64Arg (rs4994). Each gene was detected via polymerase chain reaction (PCR) using with the forward and reverse primers as follows: DIO2 forward primer $=5$ '-GGTACCATTGCCACTGTTGTCA-3', DIO2 reverse primer $=5^{\prime}$ GTCAGGTGAAATTGGGTGAGGAT-3', UCP1 forward primer = 5'-CCAGTGGTGGCTAATGAGAGAA-3', UCP1 reverse primer $=5^{\prime}$-GCACAAAGAAGAAGCAGAGAGG-3', 33 AR forward primer $=5^{\prime}$ CGCCCAATACCGCCAACAC-3', B3AR reverse primer = 5'-CCACCAGGAGTCCCATCACC-3'.

Genotyping was performed by using ABI 7500 Fast Real-time PCR System (Applied Biosystems, Foster City, CA, USA), TaqMan ${ }^{\circledR}$ Genotyping PCR Master Mix (Thermo Fisher Scientific), and TaqMan $® S N P$ Genotyping Assay (Thermo Fisher Scientific).

Biochemical analysis

Clinical data were collected as follows: sex, age at first visit, height, weight, BMI, POW, serum total cholesterol (TC), high-density lipoprotein cholesterol (HDLC), Low-density lipoprotein cholesterol (LDLC), triglycerides (TGs), random blood glucose (RBG), insulin, and hemoglobin A1c (HbA1c). Blood samples were randomly collected.

Statistical analysis

Clinical and laboratory data were summarized as median (P25-P75) for continuous variables and number (\%) for categorical variables and compared between groups stratified according to obesity status (NOB, $\mathrm{OB}$ ) and genotype prevalence using Mann-Whitney $\mathrm{U}$ test or Fisher's exact test. The association between DIO2/UCP1/ $33 A R$ genotypes and obesity was analyzed using cross tabulation and Fisher's exact test. Simple and multiple logistic regression analyses were performed to evaluate the contribution of gene polymorphism to obesity. For multiple logistic analysis, each genotype in each gene was included as an independent variable: DIO2 Thr/Thr, DIO2 Thr/Ala, DIO2 Ala/Ala, UCP1 AA, UCP1 AG, UCP1 GG, B3AR Trp/Trp, B3AR Trp/Arg, and B3AR Arg/Arg. In multiple logistic analysis, a backward stepwise selection method was applied and the best-fit model was determined according to the Akaike information criterion. In this exploratory study, sample size was not determined statistically, and multiplicity adjustment was not considered in the statistical analysis. A P value less than 0.05 was considered statistically significant. All statistical analyses were performed using SPSS version 26.0 (IBM, Armonk, NY, USA).

\section{Results}

Clinical characteristics of the subjects 
The clinical characteristics of the NOB and OB groups classified based on POW are shown in Table 1. The median age and height of the NOB group were higher than those of the OB group. For lipid profiles, TC, LDLC, and TG levels in the OB group were significantly higher than those of the NOB group, and the HDLC level in the NOB group was significantly higher than that of the OB group. For glucose metabolism parameters, $\mathrm{RBG}$, insulin, and $\mathrm{HbA} 1 \mathrm{c}$ levels in the $\mathrm{OB}$ group were significantly higher compared to those of the NOB group. Similar results were obtained from the obesity classification using Rohrer's index (Table 2).

Genotype distributions of SNPS

Genotype distributions of DIO2 Thr92Ala, UCP1 -3826 A/G, and B3AR Trp64Arg in the NOB and OB groups are shown in Table 3. The prevalence of the homozygous Ala allele of DIO2 in the OB group is significantly higher than that of the NOB group on the Thr/Thr and Ala/Ala genotype $(P=0.004)$. The genotype distribution of UCP1-3826 A/G and B3AR Trp64Arg did not significantly differ between the obese and non-obese groups ( $P=0.156$ and 0.793 , respectively). The outcomes of simple and multiple logistic analyses with respect to obesity are summarized in Table 4. DIO2 Ala/Ala was associated with obesity, with an odds ratio (OR) of $3.393(95 \% \mathrm{Cl} 1.498-7.687, P=0.003)$ via simple logistic analysis. In contrast, ORs of UCP1-3826 A/G and B3AR Trp64Arg were $1.831(95 \% \mathrm{Cl}, 0.955-3.512, P=0.069)$ and $0.819(95 \% \mathrm{Cl}, 0.263-2.555, P=0.731)$, respectively. A simple logistic analysis of obesity classification was also performed based on Rohrer's index. The results also showed that DIO2 Ala/Ala was associated with obesity, which was classified based on Rohrer's index (OR of 3.149, 95\% Cl 1.396-7.103, $P=0.006$ ) (Table 5). Multiple logistic analysis also showed that DIO2 Ala/Ala was significantly associated with obesity (Table 4). The percentage of correctly classified cases, sensitivity, and specificity of logistic analyses were $0.551(0.497-0.603), 0.628$, and 0.526 , respectively.

Clinical characteristics of each SNP genotype

Table 6 shows the clinical characteristics of DIO2 Thr92Ala. BMI, POW, and insulin levels in the DIO2 Ala/Ala genotype were significantly higher than those in the DIO2 Thr/Thr $(P<0.05)$. However, insulin levels were not significantly different after adjusting for body weight. TC, LDLC, and HbA1c of the AG genotype were significantly higher than those of the AA genotype $(P<0.05)$. Interestingly, those of the GG genotype were not significantly different. $\beta 3 A R, B M I$, and RBG of the Arg/Arg genotype were significantly lower than those of Trp/Trp (Supplemental Table 1).

\section{Discussion}

Obesity is a highly complex heterogeneous disease caused by multiple environmental, lifestyle, and genetic factors. Results from family and twin studies have suggested that genetic factors explain 40$70 \%$ of the inter-individual variation in obesity susceptibility (15). Individuals with pediatric obesity frequently remain obese in adulthood. It is important to elucidate the interaction between genetic factors and pediatric obesity, and to manage obesity in childhood. Recently, BAT has been identified as a novel target of obesity, as it increases energy expenditure by non-shivering thermogenesis. BAT-related gene 
polymorphisms are associated with obesity (8-11). However, these relationships have not yet been fully elucidated, especially in pediatric obesity. Therefore, we carried out a case-control study to investigate the association of pediatric obesity with three SNPs (DIO2 Thr92Ala, UCP1-3826 A/G, B3AR Trp64Arg) related to BAT. We demonstrated a significant correlation between the DIO2 Thr92Ala polymorphism and childhood obesity.

In the present case-control study, the frequency of the homozygous Ala allele of DIO2 was significantly higher in the OB than in the NOB group ( $15.1 \%$ vs. $6.3 \%, P=0.004)$ (Table 2$)$. These frequencies resulted in an OR of $3.393(95 \% \mathrm{Cl} 1.498-7.687)$ for the DIO2 Ala/Ala genotype in the OB group (Table 3). More than $40 \%$ of subjects with DIO2 Ala/Ala were obese, which was the highest prevalence of obesity among all genotypes (Fig. 2). This result is similar to that of a previous study showing that the DIO2 Ala/Ala genotype is strongly associated with obesity, concomitant with insulin resistance in a large cohort of patients with type 2 diabetes mellitus (8). Individuals with DIO2 Ala/Ala tend to have insulin resistance with a lower glucose disposal rate in adult obese Caucasians (11). There are three isoforms of DIO; DIO1, DIO2, and DIO3. DIO1 and DIO2 convert T4 to T3 by catalyzing 5'-deiodination, and DIO1 and DIO3 convert T4 to an inactive metabolite, rT3, by catalyzing 5-deiodination $(16,17)$. DIO2 exhibits a higher catalytic capacity than DIO1; thus, DIO2 plays a critical role in the production of T3 $(18,19)$. TH (T4 and T3) regulates metabolic processes, such as body weight, lipolysis, and thermogenesis (20). DIO2 Ala mutation impairs DIO2-mediated conversion of T4 to T3 in thyroid-deficient patients $(7,21-24)$, and DIO2 Ala mutation reduces intracellular conversion of T4 to T3 compared to DIO2 WT (23). Therefore, DIO2 Ala mutation could reduce the TH-mediated thermogenesis of BAT, leading to obesity. However, not all studies examining this genotype have verified the association with obesity, despite subjects having insulin resistance $(25,26)$. This discrepancy with the present study might be due to differences in age. In the present study, we enrolled young people aged $\leq 18$ years. In contrast, all previous studies enrolled older people aged more than 40 years. BAT mass is highly present in infancy and declines in adulthood (27). Therefore, BAT might impact childhood more than adulthood. Furthermore, the activity of BAT increases during childhood, reaching its peak at approximately 12 years, and then declines during adulthood (28). The average age of children enrolled in the present study was 12 years. This concordance in age might have affected the results. We believe that adolescents approximately 12 years of age are suitable for assessing the association of obesity and gene polymorphisms related to BAT, because other factors, such as environmental, lifestyle, and genetic factors, are likely to be more involved in the pathogenesis of obesity in adulthood. In contrast, in adulthood, insulin resistance might be the main phenotype caused by DIO2 polymorphism. DIO2 is also expressed in skeletal muscles, and TH upregulates the expression of glucose transporter 4 , which is responsible for glucose uptake. Therefore, skeletal muscle is the main glucose-consuming tissue. DIO2 polymorphism might lead to insulin resistance in adulthood, as skeletal muscle mass in adulthood is more abundant than in children. More importantly, there were no significant differences in insulin levels between DIO2 genotypes after adjusting for body weight. Higher insulin levels in the DIO2 Ala/Ala genotype might be secondary to obesity.

Previous studies have demonstrated that DIO2, UCP1, and $\beta 3 A R$ have a mutual impact on obesity in adulthood $(10,11)$. Therefore, in the present study, we examined the gene-gene interaction of the DIO2 
Thr92Ala genotype with UCP1-3826 A/G and B3AR Trp64Arg in pediatric obesity. We found no significant association between DIO2 Thr92Ala and UCP1-3826 A/G and B3AR Trp64Ar in pediatric obesity. This discrepancy from studies investigating adult obesity might also be due to age. The combination of UCP1 and $\beta 3 A R$ polymorphisms is related to reduced BAT mass with age (29), suggesting that aging uncovers the effects of the gene combination on obesity. Further studies are needed to investigate the effect of UCP1 and/or the combination of DIO2 and UCP1 on pediatric obesity.

There are a number of limitations in the present study. 1) Due to its exploratory nature, the sample size was not determined on a statistical basis, and multiplicity of tests was not considered in statistical analysis. 2) There is difference in mean age between the NOB and OB groups. The mean height is expected to increase with age; the taller children are, the higher are their BMls. 3) There is a lack of data on pubertal stage. Data on the pubertal stage are important for assessing insulin levels, as children physiologically develop insulin resistance and insulin levels increase with age, during puberty. The OB group had a higher insulin concentration, even though the median age in the OB group (11.1 years (9.113.8)) was younger than that in the NOB group (13.5 years (12.9-14.1) ). Therefore, we believe that higher insulin levels in the DIO2 Ala/Ala genotype represent insulin resistance (30). 4) Puberty alters body composition differently according to sex. During puberty, boys gain only lean mass, but girls gain both lean mass and fat mass. We cannot assess the effect of altered body composition by puberty due to a lack of data on the pubertal stage. 5) Some longitudinal studies have shown that girls have a significant decline in physical activity compared to boys during puberty $(31,32)$. This decline in physical activity due to sex difference during puberty might affect obesity. 6) We used random glucose and insulin levels in the present study. However, fasting glucose and insulin levels should be used to assess insulin resistance. 7) We included population-based non-obese children as controls in the present study. However, we should have enrolled hospital-based non-obese children as controls to align the background of the participants. Due to the various limitations of this study, further studies are needed to validate the results.

In conclusion, our results indicate that the homozygous Ala/Ala allele of the DIO2 gene is associated with an increased risk of obesity and insulin resistance in children. The present study also suggests that pediatric obesity might be suitable for assessing the association with gene polymorphisms related to BAT. These findings advance our understanding of the genetic contribution to the pathogenesis of obesity and might develop new strategies against obesity. However, this is an exploratory study; thus, further studies, including a genome wide association study, are needed to validate these findings.

\section{Declarations}

\section{Ethics approval and consent to participate}

This study was approved by the Ethics Committee of the Kyoto Prefectural University of Medicine. Written informed consent was obtained from each patient and their parents.

\section{Consent for publication}


Written informed consent for the publication of clinical details and images was obtained from the patient's parents. A copy of the consent form is available for review by the Editor of this journal.

\section{Availability of data and material}

The datasets supporting the conclusions of this article are included within the article.

\section{Competing interests}

Each author declares that they have no competing interests.

\section{Funding}

This work was supported by JSPS grant numbers 24650427 and $15 \mathrm{~K} 01686$.

\section{Authors' contributions}

J.M., M.Y., S.M., Y.K., H.M., K.K., S.S., K.K., and H.N. attended to the patients; T.O., Y.T., K.S., S.F and K.K. performed the gene analysis; T.O., J.M., H.Y. and H.N. analyzed the data; T.O. and J.M. wrote the manuscript; J.M., K.K., H.N., and H.H. provided conceptual advice. All authors have read and approved the final manuscript.

\section{References}

1. Haslam DW, James WP. Obesity. Lancet. 2005;366:1197-209.

2. Maes HH, Neale MC, Eaves LJ. Genetic and environmental factors in relative body weight and human adiposity. Behav Genet. 1997;27:325-51.

3. Tokgöz Y, Işık IA, Akbari S, Kume T, Sayın O, Erdal E, Arslan N. Perilipin polymorphisms are risk factors for the development of obesity in adolescents? A case-control study. Lipids Health Dis. 2017;16:52.

4. Morimoto H, Mori J, Nakajima H, Kawabe Y, Tsuma Y, Fukuhara S, Kodo K, Ikoma K, Matoba S, Oudit GY, Hosoi H. Angiotensin 1-7 stimulates brown adipose tissue and reduces diet-induced obesity. Am J Physiol Endocrinol Metab. 2018;314:E131-8.

5. Kawabe Y, Mori J, Morimoto H, Yamaguchi M, Miyagaki S, Ota T, Tsuma Y, Fukuhara S, Nakajima H, Oudit GY, Hosoi H. ACE2 exerts anti-obesity effect via stimulating brown adipose tissue and induction of browning in white adipose tissue. Am J Physiol Endocrinol Metab. 2019;317:E1140-9.

6. Kodo K, Sugimoto S, Nakajima H, Mori J, Itoh I, Fukuhara S, Shigehara K, Nishikawa T, Kosaka K, Hosoi H. Erythropoietin (EPO) ameliorates obesity and glucose homeostasis by promoting thermogenesis and endocrine function of classical brown adipose tissue (BAT) in diet-induced obese mice. PLoS One. 2017;12:e0173661.

7. Cioffi F, Gentile A, Silvestri E, Goglia F, Lombardi A. Effect of lodothyronines on Thermogenesis: Focus on Brown Adipose Tissue. Front Endocrinol (Lausanne). 2018;9:254. 
8. Dora JM, Machado WE, Rheinheimer J, Crispim D, Maia AL. Association of the type 2 deiodinase Thr92Ala polymorphism with type 2 diabetes: case-control study and meta-analysis. Eur $\mathrm{J}$ Endocrinol. 2010;163:427-34.

9. Chen Y, Wang X, Shen Z, Fan P, Liu R, Liu Y, Ren R, Ma L, Bai H. Effect of the beta-3 adrenergic receptor Trp64Arg and uncoupling $1-3826$ A > G genotypes on lipid and apolipoprotein levels in overweight/ obese and non-obese Chinese subjects. Lipids Health Dis. 2015;14:34.

10. Hayakawa T, Nagai Y, Taniguchi M, Yamashita H, Takamura T, Nomura G, Kobayashi K. Phenotypic characterization of the beta3-adrenergic receptor mutation and the uncoupling protein 1 polymorphism in Japanese men. Metabolism. 1999;48:636-40.

11. Mentuccia D, Proietti-Pannunzi L, Tanner K, Bacci V, Pollin TI, Poehlman ET, Shuldiner AR, Celi FS. Association between a novel variant of the human type 2 deiodinase gene Thr92Ala and insulin resistance: evidence of interaction with the Trp64Arg variant of the beta-3-aderenergic receptor. Diabetes. 2002;51:880-3.

12. The Japan Society for the Study of Obesity. Guidelines for the management of obesity disease in children and adolescents 2017. The judgement for childhood obesity. Tokyo (Japan): Life Science publishing. 2017. P. 3-4.

13. Isojima T, Yokoya S. The value of anthrometric indices for childhood obesity in Japan. Ann Hum Biol. 2019;46:293-7.

14. Dobashi K. Evaluation of obesity in school-age children. J Atheroscler Thromb. 2016;23:32-8.

15. Maes HH, Neale MC, Eaves LJ. Genetic and environmental factors in relative body weight and human obesity. Behavioral Genetics. 1997;27:325-51.

16. Germain DL, Galton VA. The deiodinase family of selenoproteins. Thyroid. 1997;7:655-8.

17. Luongo $C$, Dentice $M$, Salvatore $D$. Deiodinases and their intricate role in thyroid hormone homeostasis. Nat Rev Endocrinol. 2019; 479 - 88.

18. Maia AL, Kim BW, Huang SA, Harney JW, Larsen PR. Type 2 iodothyronine deiodinase is the major source of plasma T3 in euthyroid humans. J Clin Invest. 2005;115:2524-33.

19. Luongo $C$, Dentice $M$, Salvatore $D$. Deiodinases and their intricate role in thyroid hormone homeostasis. Nat Rev Endocrinol. 2019;15:479-88.

20. Mullur R, Liu YY, Brent GA. Thyroid hormone regulation of metabolism. Physiol Rev. 2014;94:355-82.

21. He B, Li J, Wang G, Ju W, Lu Y, Shi Y, HeL, Zhong N. Association of genetic polymorphism in the type Il deiodinase gene with bipolar disorder in a subset of Chinese population. Prog Neuropsychopharmacol Biol Psychiatry. 2009;33:986-90.

22. Butler PW, Smith SM, Linderman JD, Brychta RJ, Alberobello AT, Dubaz OM, Luzon JA, Skarulis MC, Cochran CS, Wesley RA, Pucino F, Celi FS. The Thr92Ala 5' type 2 deiodinase gene polymorphism is associated with a delayed triiodothyronine secretion in response to the thyrotropin-releasing hormone-stimulation test: a pharmacogenomic study. Thyroid. 2010;20:1407-12. 
23. Castagna MG, Dentice M, Cantara S, Ambrosio R, Maino F, Porcelli T, Marzocchi C, Garbi C, Pacini F, Salvatore D. DIO2 Thr92Ala reduces deiodinase-2 activity and serum-T3 levels in thyroid-deficient patients. J Clin Endocrinol Metab. 2017;102:1623-30.

24. Cantara S, Ricci C, Maino F, MarzocchiC, Pacini F, Castagna MG. Variants in MCT10 protein do not affect FT3 levels in athyreotic patients. Endocrine. 2019;66:551-6.

25. Maia AL, Dupuis J, Manning A, Liu C, Meigs JB, Cupples LA, Larsen PR, Fox CS. The type 2 deiodinase (DIO2) A/G polymorphism is not associated with glycemic traits: the Framingham Heart Study. Thyroid. 2007;17:199-202.

26. Grarup N, Andersen MK, Andreasen $\mathrm{CH}$, Albrechtsen A, Borch-Johnsen K, Jorgensen T, Auwerx J, Schmitz O, Hansen T, Pedersen O. Studies of the common DIO2 Thr92Ala polymorphism and metabolic phenotypes in 7342 Danish white subjects. J Clin Endocrinol Metab. 2007;92:363-6.

27. Enerback S. Human brown adipose tissue. Cell Metab. 2010;11:248-52.

28. Gilsanz V, Chung SA, Jackson H, Dorey FJ, Hu HH. Functional brown adipose tissue is related to muscle volume in children and adolescents. J Pediatr. 2010;158:722-6.

29. Yoneshiro T, Ogawa T, Okamoto N, Matsushita M, Aita S, Kameya T, Kawai Y, Iwanaga T, Saito M. Impact of UCP1 and $\beta A R$ gene polymorphisms on age-related changes in brown adipose tissue and adiposity in humans. Int J Obes (Lond). 2013;37:993-8.

30. Kelsey MM, Zeitler PS. Insulin Resistance of Puberty. Curr Diab Rep. 2016;16:64.

31. Basterfield L, Adamson AJ, Frary JK, Parkinson KN, Pearce MS, Reilly JJ, Gateshead Millennium Study Core Team. Longitudinal study of physical activity and sedentary behavior in children. Pediatrics. 2011;127:e24-30.

32. Nader PR, Bradley RH, Houts RM, McRitchie SL, O'Brien M. Moderate-to-vigorous physical activity from ages 9 to 15 years. JAMA. 2008;300:295-305.

\section{Tables}

Table 1. Clinical characteristics in the non-obese and obese children group, classified based on POW 


\begin{tabular}{lccc}
\hline & NOB $(\mathrm{n}=270)$ & OB $(\mathrm{n}=86)$ & $P$ \\
\hline POW $(\%)$ & $-4.4(-11.3-2.9)$ & $45.9(31.7-57.2)$ & $<0.001$ \\
A ge (years) & $13.5(12.9-14.1)$ & $11.1(9.1-13.8)$ & $<0.001$ \\
Gender male/ female & $144 / 126$ & $44 / 42$ & 0.804 \\
Height $(\mathrm{cm})$ & $155.8(150.7-161.1)$ & $146.7(135.5-156.6)$ & $<0.001$ \\
Body weight $(\mathrm{kg})$ & $45.1(40.3-50.8)$ & $56.1(46.1-67.5)$ & $<0.001$ \\
BMI $(\mathrm{kg} / \mathrm{m} 2)$ & $18.5(17.3-19.7)$ & $25.5(24.0-28.1)$ & $<0.001$ \\
TC $(\mathrm{mg} / \mathrm{dL})$ & $169.0(152.0-186.0)$ & ${ }^{\mathrm{c}} 173.0(159.0-198.0)$ & 0.021 \\
HDLC $(\mathrm{mg} / \mathrm{dL})$ & $66.0(58.0-75.0)$ & ${ }^{\mathrm{c}} 52.0(44.0-62.0)$ & $<0.001$ \\
LDLC $(\mathrm{mg} / \mathrm{dL})$ & $88.5(73.0-106.0)$ & ${ }^{\mathrm{c}} 104.0(86.0-123.0)$ & $<0.001$ \\
TG $(\mathrm{mg} / \mathrm{dL})$ & $74.5(53.0-112.0)$ & ${ }^{\mathrm{c}} 135.0(90.0-181.0)$ & $<0.001$ \\
RBG $(\mathrm{mg} / \mathrm{dL})$ & ${ }^{\mathrm{a}} 85.0(80-89.5)$ & $91.0(85-98)$ & $<0.001$ \\
Insulin $(\mu \mathrm{IU} / \mathrm{mL})$ & ${ }^{\mathrm{b}} 5.75(3.80-9.60)$ & ${ }^{\mathrm{d}} 16.30(9.91-31.40)$ & $<0.001$ \\
HbA $1 \mathrm{c}(\%)$ & $5.2(5.1-5.3)$ & ${ }^{\mathrm{e}} 5.4(5.2-5.6)$ & $<0.001$ \\
\hline
\end{tabular}

Data are presented as medians (P25-P75) and n. NOB, non-obese children group; OB, obese children group; BMI, body mass index; POW, percentage of overweight; TC, total cholesterol; HDLC, high-density lipoprotein cholesterol; LDLC, low-density lipoprotein cholesterol; TG, triglyceride; RBG, random blood glucose; HbA1c, hemoglobin A1c. ${ }^{a} n=268,{ }^{b} n=239,{ }^{c} n=85,{ }^{d} n=82,{ }_{n}=80$

Table 2. Clinical characteristics in the non-obese and obese children group, classified based on the Rohrer's index

\begin{tabular}{lccc}
\hline & NOB $(\mathrm{n}=265)$ & OB $(\mathrm{n}=91)$ & $P$ \\
\hline Rohrer's index & $117.7(110.1-127.7)$ & $181.9(165.6-197.9)$ & $<0.001$ \\
A ge (years) & $13.5(12.9-14.1)$ & $11.2(9.4-13.8)$ & $<0.001$ \\
Gender male/ female & $142 / 123$ & $46 / 45$ & 0.628 \\
Height $(\mathrm{cm})$ & $155.8(150.7-161.2)$ & $149.0(135.5-156.6)$ & $<0.001$ \\
Body weight $(\mathrm{kg})$ & $45.0(40.2-50.4)$ & $55.6(47.5-67.4)$ & $<0.001$ \\
BMI $(\mathrm{kg} / \mathrm{m} 2)$ & $18.5(17.3-19.7)$ & $25.4(23.5-28.1)$ & $<0.001$ \\
TC $(\mathrm{mg} / \mathrm{dL})$ & $169.0(151.0-186.0)$ & ${ }^{\mathrm{c}} 173.0(159.0-197.0)$ & 0.020 \\
HDLC $(\mathrm{mg} / \mathrm{dL})$ & $66.0(58.0-75.0)$ & ${ }^{\mathrm{c}} 53.0(44.0-63.0)$ & $<0.001$ \\
LDLC $(\mathrm{mg} / \mathrm{dL})$ & $88.0(73.0-106.0)$ & ${ }^{\mathrm{c}} 102.0(87.0-123.0)$ & $<0.001$ \\
TG $(\mathrm{mg} / \mathrm{dL})$ & $75.0(54.0-111.0)$ & ${ }^{\mathrm{c}} 132.5(83.0-181.0)$ & $<0.001$ \\
RBG $(\mathrm{mg} / \mathrm{dL})$ & ${ }^{\mathrm{a}} 85.0(80.0-90.0)$ & $90.0(85.0-96.0)$ & $<0.001$ \\
Insulin $(\mu \mathrm{IU} / \mathrm{mL})$ & ${ }^{\mathrm{b}} 5.75(3.80-9.57)$ & ${ }^{\mathrm{d}} 15.70(9.86-29.22)$ & $<0.001$ \\
HbA $1 \mathrm{c}(\%)$ & $5.2(5.1-5.3)$ & ${ }^{\mathrm{e}} 5.4(5.2-5.5)$ & $<0.001$ \\
\hline
\end{tabular}

Data are presented as medians (P25-P75) and n. NOB, non-obese children group; OB, obese children group; BMI, body mass index; TC, total cholesterol; HDLC, high-density lipoprotein cholesterol; LDLC, low- 
density lipoprotein cholesterol; TG, triglyceride; RBG, random blood glucose; HbA1c, hemoglobin A1c. ${ }^{a} \mathrm{n}=$ $263,{ }^{b} n=235,{ }^{c} n=90,{ }^{d} n=86, e_{n}=85$

Table 3. Genotype distributions of SNPs

\begin{tabular}{cccc}
\hline & NOB $(\mathrm{n}=270)$ & OB $(\mathrm{n}=86)$ & $P$ \\
\hline DIO2 Thr92Ala & & & 0.008 \\
Thr/Thr & $0.524(142)$ & $0.376(32)$ & \\
Thr/Ala & $0.410(111)$ & $0.482(41)$ & \\
Ala/Ala & $0.063(17)$ & $0.151(13)^{*}$ & \\
\hline UCP1 -3826 A/G & & & \\
AA & $0.348(94)$ & $0.244(21)$ & \\
AG & $0.407(110)$ & $0.442(38)$ & \\
GG & $0.244(66)$ & $0.314(27)$ & \\
\hline B3ADR Trp64Arg & & & \\
Trp/Trp & $0.656(177)$ & $0.628(54)$ & \\
Trp/Arg & $0.285(77)$ & $0.326(28)$ & \\
Arg/Arg & $0.059(16)$ & $0.047(4)$ & \\
\hline
\end{tabular}

Numbers in the parentheses indicate the number of the subjects. *Compared to the NOB group on the Thr/Thr and Ala/Ala genotype $(P=0.004)$.

Table 4. Univariate and multivariate analyses with respect to obesity classified based on percentage of weight

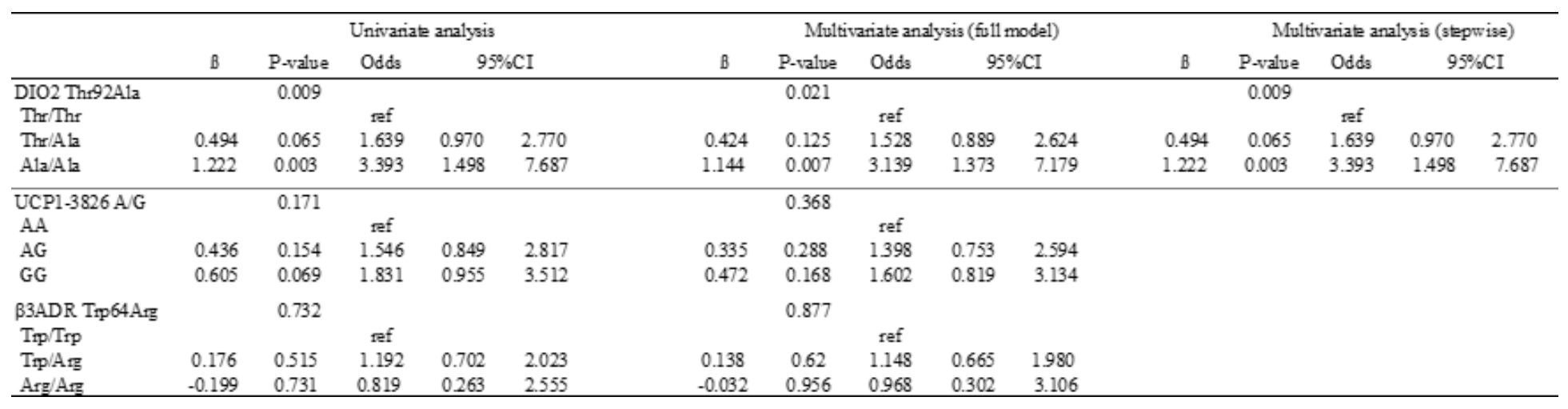

Table 5. Univariate and multivariate analyses with respect to obesity

classified based on Rohrer's index 


\begin{tabular}{lccccc}
\hline & \multicolumn{5}{c}{ Univariate analysis } \\
\cline { 2 - 6 } & $\beta$ & P-value & Odds & $95 \% \mathrm{CI}$ \\
\hline DIO2 Thr92Ala & & 0.009 & & & \\
Thr/Thr & & & ref & \\
Thr/Ala & 0.494 & 0.065 & 1.639 & 0.970 & 2.770 \\
Ala/Ala & 1.222 & 0.003 & 3.393 & 1.498 & 7.687 \\
& & & & & \\
\hline UCP1-3826 A/G & & 0.171 & & & \\
AA & & & ref & & \\
AG & 0.436 & 0.154 & 1.546 & 0.849 & 2.817 \\
GG & 0.605 & 0.069 & 1.831 & 0.955 & 3.512 \\
& & & & & \\
\hline B3ADR Thr64Arg & & 0.732 & & & \\
Trp/Trp & & & ref & & \\
Trp/Arg & 0.176 & 0.515 & 1.192 & 0.702 & 2.023 \\
Arg/Arg & -0.199 & 0.731 & 0.819 & 0.263 & 2.555 \\
\hline
\end{tabular}

yUDZQNIA2UDZQNIA2UDZQNIA2UDfwUG/gN+SXHKfD9n+QAAAAASUVORK5CYII=">

Table 6. Clinical characteristics of the DIO2 Thr92Ala SNP genotype

\begin{tabular}{|c|c|c|c|}
\hline & \multicolumn{3}{|c|}{ DIO2 Thr/92Ala } \\
\hline & $\operatorname{Thr} / \operatorname{Th} r(n=174)$ & $\mathrm{Thr} / \mathrm{Ala}(\mathrm{n}=152)$ & Ala/Ala $(n=30)$ \\
\hline Age (years) & $13.6(12.9-14.2)$ & $13.1(12.4-13.9)^{*}{ }^{*} \cdot$ & $12.9(11.1-13.9)$ \\
\hline Gender male/ female & $89 / 85$ & $84 / 68$ & $15 / 15$ \\
\hline Height $(\mathrm{cm})$ & $155.4(150.2-160.9)$ & $154.5(146.1-160.2)$ & $151.4(142.0-159.5)^{*}$ \\
\hline Body weight (kg) & $46.5(40.6-52.8)$ & $46.1(40.4-52.2)$ & $49.7(42.1-56.0)$ \\
\hline BMI $\left(\mathrm{kg} / \mathrm{m}^{2}\right)$ & $19.0(17.5-20.8)$ & $19.1(17.7-22.7)$ & $21.8(19.3-26.3)^{\text {*k* }}$ \\
\hline POW (\%) & $0.2(-9.6-10.9)$ & $-0.5(-9.0-24.9)$ & $16.1(-3.3-46.4)^{* * *}$ \\
\hline Rohrer's index & $122.0(111.9-136.9)$ & $123.8(114.2-155.4)$ & $142.6(119.9-184.9)^{k * k}$ \\
\hline $\mathrm{TC}(\mathrm{mg} / \mathrm{dL})$ & $169.0(152.0-186.0)$ & ${ }^{\mathrm{d}} 170.0(154.0-192.0)$ & $176.0(163.0-193.0)$ \\
\hline $\operatorname{HDLC}(\mathrm{mg} / \mathrm{dL})$ & $64.0(55.0-73.0)$ & ${ }^{\mathrm{d}} 63.0(54.0-73.0)$ & $62.0(52.0-68.0)$ \\
\hline LDLC (mg/dL) & $89.5(73.0-108.0)$ & ${ }^{d} 93.0(78.2-112.0)$ & $96.5(84.0-109.0)$ \\
\hline TG (mg/dL) & $87.0(56.0-135.0)$ & ${ }^{\mathrm{d}} 82.0(56.0-129.0)$ & $95.5(61.0-127.0)$ \\
\hline $\mathrm{RBG}(\mathrm{mg} / \mathrm{dL})$ & ${ }^{\mathrm{a}} 86.0(80.0-90.0)$ & $87.0(80.5-93.0)$ & $86.5(83.0-90.0)$ \\
\hline Insulin $(\mu \mathrm{IU} / \mathrm{mL})$ & ${ }^{\mathrm{b}} 6.70(4.77-12.32)$ & ${ }^{\mathrm{e}} 7.59(4.13-15.60)$ & ${ }^{\mathrm{g}} 11.80(4.46-18.15)^{*}$ \\
\hline $\mathrm{HbA} 1 \mathrm{c}(\%)$ & ${ }^{\mathrm{c}} 5.2(5.1-5.4)$ & $\mathrm{f}_{5.2}(5.1-5.4)$ & ${ }^{\mathrm{h}_{5} .2(5.0-5.5)}$ \\
\hline
\end{tabular}

Data are presented as means (P25-P75) and n. BMI, body mass index; POW, percentage of overweight;

TC, total cholesterol; HDLC, high-density lipoprotein cholesterol; LDLC, low-density lipoprotein cholesterol; 
TG, triglycerides; RBG, random blood glucose; HbA1c, hemoglobin A1c. ${ }^{*} P<0.05$ vs. wild type of the corresponding gene, ${ }^{* *} P<0.01$ vs. wild type of the corresponding gene. ${ }^{a} n=172,{ }^{b} n=162,{ }^{c} n=173$, ${ }^{d} n=151,{ }^{e} n=130,{ }^{f} n=149,{ }^{g} n=29,{ }^{h} n=28$

\section{Figures}

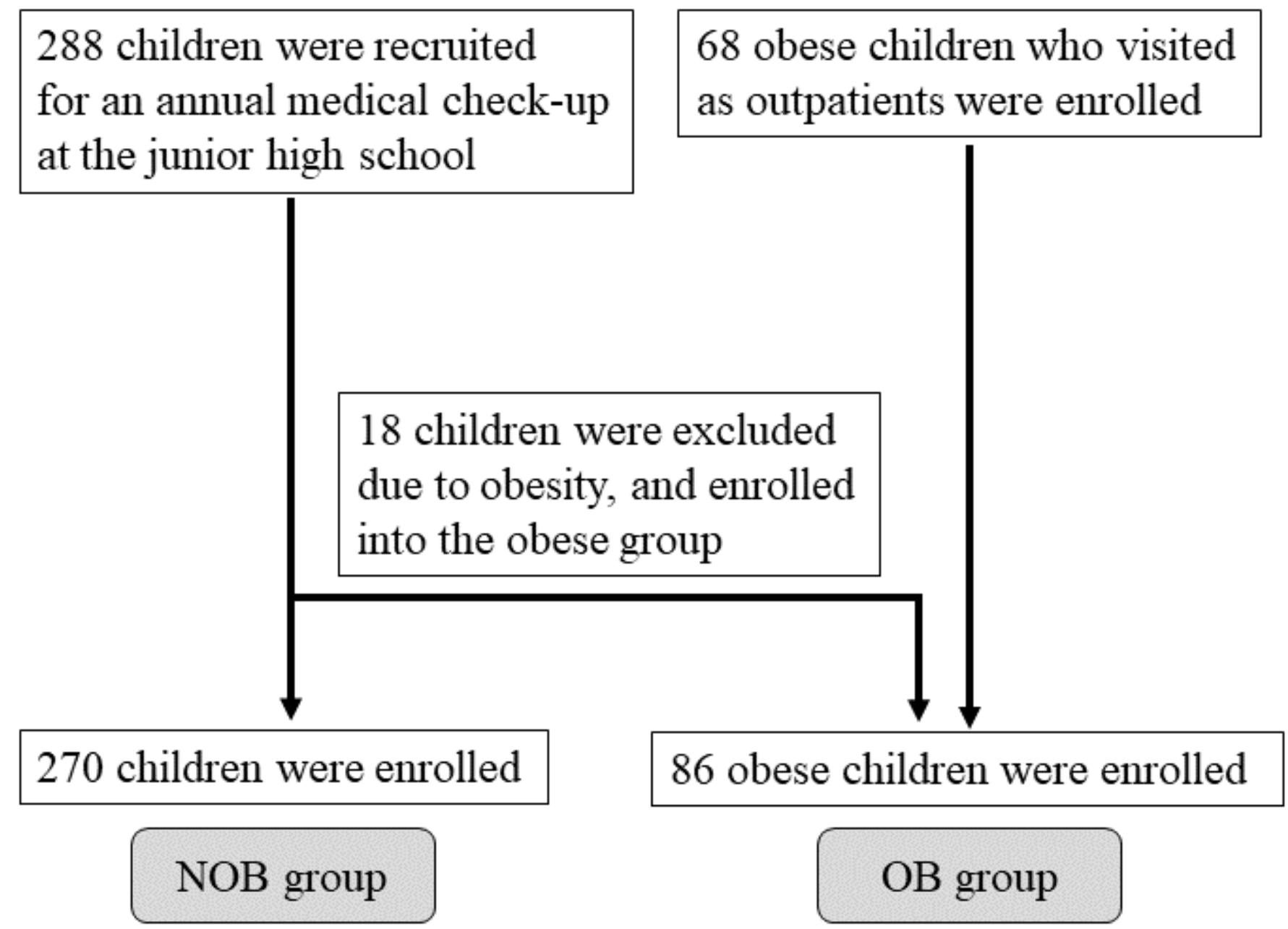

Figure 1

Flowchart for study data selection. 


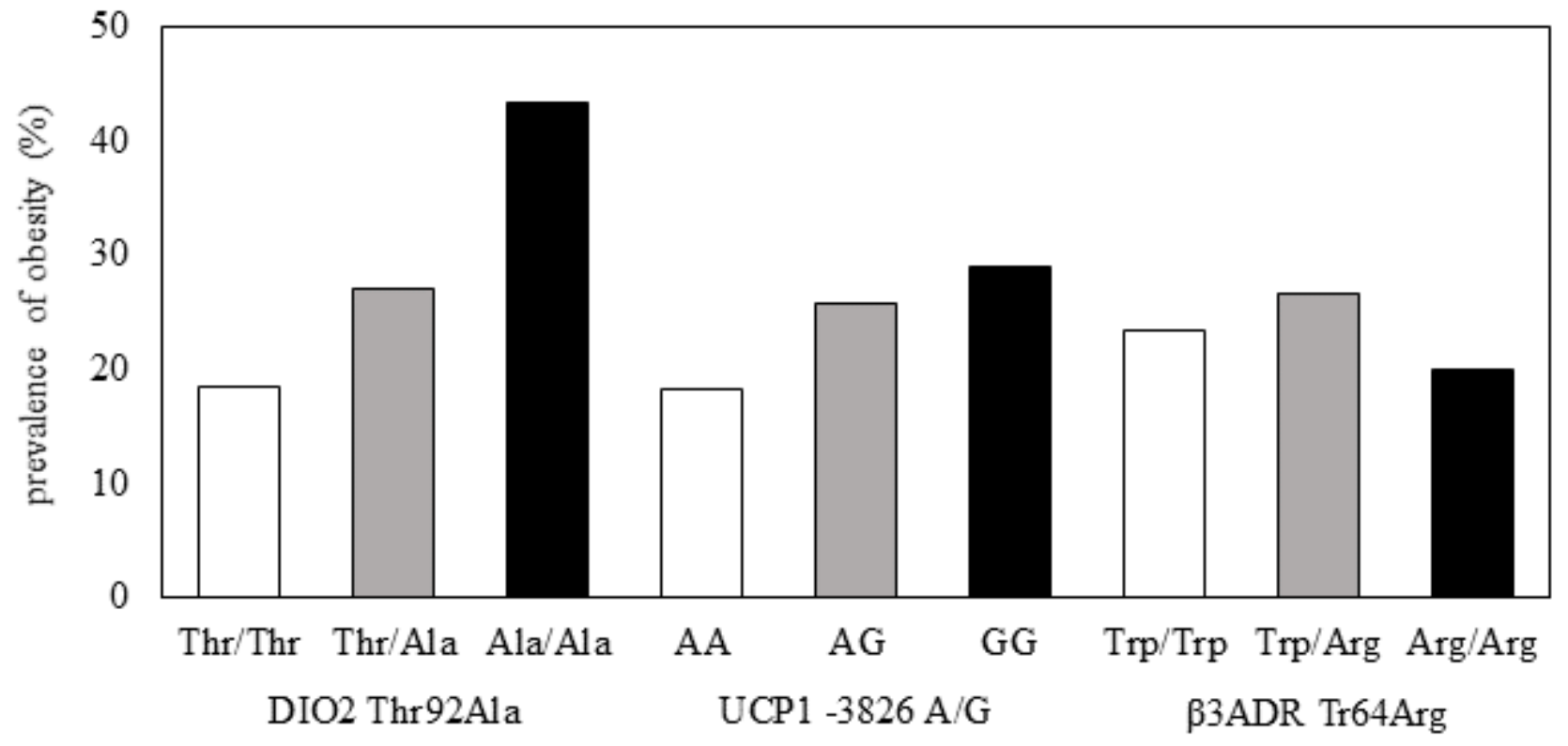

Figure 2

Correlation graph between all genotypes and the prevalence of obesity.

\section{Supplementary Files}

This is a list of supplementary files associated with this preprint. Click to download.

- SupplementalTableLHADD2000448.docx 